\title{
In Vitro Cytotoxicity Evaluation of a Novel Root Repair Material
}

\author{
Hui-min Zhou, PbD, ${ }^{*}{ }^{+}$Ya Shen, DDS, PbD, ${ }^{\dagger}$ Zhe-jun Wang, DDS, PbD, ${ }^{\dagger \neq} \mathrm{Li} \mathrm{Li}, \mathrm{PbD},{ }^{*}$ \\ Yu-feng Zheng, PbD, ${ }^{\star \downarrow}$ Lari Häkkinen, DDS, PbD, ${ }^{\|}$and Markus Haapasalo, DDS, PbD ${ }^{\dagger}$
}

\begin{abstract}
Ahstract
Introduction: This study examined the effect of a new bioactive dentin substitute material (Biodentine) on the viability of human gingival fibroblasts. Methods: Biodentine, White ProRoot mineral trioxide aggregate (MTA), and glass ionomer cement were evaluated. Human gingival fibroblasts were incubated for 1, 3, and 7 days both in the extracts from immersion of set materials in culture medium and directly on the surface of the set materials immersed in culture medium. Fibroblasts cultured in Dulbecco modified Eagle medium were used as a control group. Cytotoxicity was evaluated by flow cytometry, and the adhesion of human gingival fibroblasts to the surface of the set materials was assessed by using scanning electron microscopy. The data of cell cytotoxicity were analyzed statistically by using a one-way analysis of variance test at a significance level of $P<.05$. Results: Cells exposed to extracts from Biodentine and MTA showed the highest viabilities at all extract concentrations, whereas cells exposed to glass ionomer cement extracts displayed the lowest viabilities $(P<.05)$. There was no significant difference in cell viabilities between Biodentine and MTA during the entire experimental period $(P>.05)$. Human gingival fibroblasts in contact with Biodentine and MTA attached to and spread over the material surface after an overnight culture and increased in numbers after 3 and 7 days of culture. Conclusions: Biodentine caused gingival fibroblast reaction similar to that by MTA. Both materials were less cytotoxic than glass ionomer cement. (J Endod 2013;39:478-483)
\end{abstract}

\section{Key Words}

Biocompatibility, Biodentine, calcium silicate-based materials, cell adhesion, cytotoxicity, flow cytometry, glass ionomer, human gingival fibroblast, MTA /arious materials have been used for root repair, including silver amalgam, zinc oxide-eugenol, calcium hydroxide, composites, and glass ionomers. However, none of them are ideal for the special conditions and requirements of root repair. For instance, silver amalgam has concerns associated with it such as the possibility of mercury pollution as well as poor esthetics. Resin-containing composite materials release toxic monomers and shrink during polymerization (1). Since the introduction of mineral trioxide aggregate (MTA) (Dentsply Tulsa Dental Specialties, Tulsa, OK) into the field of root repair materials (2), calcium silicate-based materials have attracted considerable attention (3-8) owing to their superior sealing ability, biocompatibility, regenerative capabilities, and antibacterial properties (4, 6, 9-12). Thus, they have been widely used both as endodontic repair materials and as dentin substitutes (13). Several calcium silicate-based root repair materials have been developed and are available on the market such as ProRoot (Dentsply Tulsa Dental Specialties), MTA Plus (Prevest-Denpro, Jammu City, India), and Endosequence Root Repair Material Putty and Paste (Brasseler USA, Savannah, GA). However, there are some drawbacks associated with the use of the bioceramic cements including long setting time, difficult manipulation, limited resistance to washout before setting, and possibility of staining of tooth structure $(4-6,14)$. Therefore, new root repair materials are continually being developed to further improve their properties.

Recently, a new calcium silicate-based material, Biodentine (Septodont, SaintMaur-des-Fossés, France), was introduced. Biodentine is claimed by the manufacturer to possess the benefits but not the drawbacks of some other bioceramic cements. According to the manufacturer, Biodentine contains tricalcium silicate, calcium carbonate, and zirconium oxide, and a water-based liquid-containing calcium chloride as the setting accelerator and a water-reducing agent (1). It is a fast-setting calcium silicate-based restorative material recommended for use as a dentin substitute that can be used both as a coronal restoration material for perforation repair and as a pulp-capping material in direct contact with the pulp (1).

Cytotoxicity of a root filling material, when used in pulp capping, perforation repair, or as a retrograde filling, may influence the viability of periradicular cells and cause cell death by apoptosis or necrosis (15). To promote healing and restoration of the function of the tooth, dental materials should either stimulate repair or be biologically neutral (16). Therefore, it is important to avoid dental materials that are toxic to the pulpal and periapical tissues that might compromise the clinical outcome (17, 18). Currently, there is limited information available about the cytotoxicity of

From the *Center for Biomedical Materials and Engineering, College of Material Science and Chemical Engineering, Harbin Engineering University, Harbin, China; ${ }^{\dagger}$ Division of Endodontics, Department of Oral Biological and Medical Sciences, Faculty of Dentistry, University of British Columbia, Vancouver, British Columbia, Canada; ${ }^{\ddagger}$ State Key Laboratory Breeding Base of Basic Science of Stomatology (Hubei-MOST) and Key Laboratory of Oral Biomedicine Ministry of Education, School and Hospital of Stomatology, Wuhan University, Wuhan, China; ${ }^{\S}$ State Key Laboratory for Turbulence and Complex Systems and Department of Materials Science and Engineering, College of Engineering, Peking University, Beijing, China; and "Laboratory of Periodontal Biology, Department of Oral Biological and Medical Sciences, Faculty of Dentistry, University of British Columbia, Vancouver, British Columbia, Canada.

Supported by the Natural Science Foundation of Heilongjiang Province of China (no. ZD201012).

Address requests for reprints to Prof Markus Haapasalo, Chair, Division of Endodontics, Head, Department of Oral Biological and Medical Sciences, UBC Faculty of Dentistry, 2199 Wesbrook Mall, Vancouver, BC, Canada V6T 1Z3. E-mail address: markush@dentistry.ubc.ca

0099-2399/\$ - see front matter

Copyright @ 2013 American Association of Endodontists.

http://dx.doi.org/10.1016/j.joen.2012.11.026 
Biodentine to periodontal cells $(1,19-22)$. Therefore, the purpose of this study was to evaluate the cytotoxicity of Biodentine to human gingival fibroblasts in comparison with MTA and glass ionomer cements.

\section{Materials and Methods}

Two commercially available calcium silicate-based cements, Biodentine (batch \#B01647) and White ProRoot MTA (batch \#11004158), and a glass ionomer cement (GIC) (GC Fuji IX GP, batch \#1111021; GC Corporation, Tokyo, Japan) were used in the present study.

\section{Cell Culture}

Human gingival fibroblasts were used to evaluate the biological response of the 3 types of cement. Biocompatibility parameters included cell viability as measured by flow cytometry and cell morphology as studied by scanning electron microscopy (SEM). Human gingival fibroblasts were obtained from previously established stocks cultured from healthy patients who underwent oral surgery (23). Fibroblasts of the seventh to eighth passage were used for the experiments. Standard protocols were followed in establishing and maintaining the cultures. Dulbecco modified Eagle medium (DMEM) (Gibco, Grand Island, NY) supplemented with $100 \mu \mathrm{g} / \mathrm{mL}$ penicillin G, $50 \mu \mathrm{g} / \mathrm{mL}$ streptomycin, $0.25 \mu \mathrm{g} / \mathrm{mL}$ Fungizone, and $10 \%$ fetal bovine serum (Gibco) was used as the cell culture medium.

\section{Preparation of Cement Elutes}

Biodentine, MTA, and GIC specimens were shaped with 3-mmthick rubber molds with a diameter of $10 \mathrm{~mm}$, according to the manufacturers' instructions under aseptic conditions. Three samples of each material were allowed to set at $37^{\circ} \mathrm{C}$ in $100 \%$ relative humidity for 2 days. After setting, the disks were exposed to ultraviolet light for 20 minutes on each surface to ensure sterility and transferred into 24well tissue culture plates containing $1 \mathrm{~mL}$ DMEM per well. The surface area to volume ratio used for extract preparation was about $250 \mathrm{~mm}^{2}$ / $\mathrm{mL}$ in accordance with ISO standard 10993-5 (24). One milliliter of extract was drawn from each well after incubation at $37^{\circ} \mathrm{C}$ and $100 \%$ relative humidity for 24 hours. Each extract was divided into 5 aliquots to obtain 5 parallel experimental groups. The extracts were serially diluted 1:1 with DMEM to achieve a total of 5 concentrations of each extract. DMEM without the materials incubated for 24 hours was used as control.

\section{Flow Gytometry}

A quantitative flow cytometry test was performed after each day of culture to characterize cell viability. Cells were plated at a density of 5000 cells $/ \mathrm{cm}^{2}$ in a 24-well plate and incubated with or without different concentrations of extracts diluted in DMEM for 1, 3, and 7 days. After culture for the indicated time, cells from different test groups and controls were washed twice with phosphate-buffered saline (PBS) (Sigma-Aldrich, St Louis, MO) and detached from the culture wells with $0.25 \%$ trypsin. The collected cells were centrifuged to discard the supernatant and resuspended at $1 \times 10^{4}$ cells $/ \mathrm{mL}$ in PBS. The cells were stained with fluorescein calcein $\mathrm{AM}\left(\lambda_{\mathrm{abs}} / \lambda_{\mathrm{em}}=494 / 517 \mathrm{~nm}\right.$, green fluorescence) and ethidium homodimer-1 (EthD-1) $\left(\lambda_{\mathrm{abs}} /\right.$ $\lambda_{\mathrm{em}}=528 / 617 \mathrm{~nm}$, red fluorescence) according to the flow cytometry protocol for a viability assay (live/dead viability/cytotoxicity kit for mammalian cells; Molecular Probes Inc, Eugene, OR) and incubated for 20 minutes at room temperature, while protected from light. The stained cells were analyzed by flow cytometer (BD FACSCalibur; BD Biosciences, Franklin Lakes, NJ) by using 488-nm excitation and measuring green fluorescence emission for calcein and red fluorescence emission $(617 \mathrm{~nm})$ for EthD-1. Single-color-stained cells and nonstained cells were used to standardize the settings and determine background autofluorescence, respectively. The percentage distributions of viable and dead cells were determined by FlowJo software (Tree Star, Inc, Ashland, OR). Experiments were performed in triplicate.

\section{Cell Adhesion Assay}

Cell adhesion on the surface of materials after culture for 1, 3, and 7 days was observed by SEM. The specimens of Biodentine, MTA, and GIC were shaped into disks of $5 \mathrm{~mm}$ in diameter and $1.6 \mathrm{~mm}$ in thickness by using rubber molds under the same conditions as the cytotoxicity assay. Twenty-five disks of each material were prepared and subdivided into 5 groups (A-E). Each group contained 5 parallel samples $(n=5)$. To remove the toxic by-products, all disks were first incubated at $37^{\circ} \mathrm{C}$ in the wells of 24-well tissue culture plates (Sarstedt, Inc, Montreal, Canada) containing $1 \mathrm{~mL}$ distilled water that was changed daily for 5 days. Group A comprised disks that had been incubated in distilled water only. In group B, 1 mL DMEM without cells was added to each well containing a disk and incubated for 7 days. In groups C-E, the disks were incubated in DMEM culture medium for 7 days and then seeded with gingival fibroblasts $\left(5 \times 10^{4}\right.$ cells/well with 1 mL DMEM) for 1 day (group C), 3 days (group D), and 7 days (group E), respectively.

Specimens for SEM examination were prefixed with phosphatebuffered $2.5 \%$ glutaraldehyde (Sigma-Aldrich) for 30 minutes before further fixation in $1 \%$ osmium tetroxide $\left(\mathrm{OsO}_{4}\right)$ for 1 hour. The specimens were subsequently rinsed in PBS and dehydrated in sequentialgraded concentrations of ethanol $(50 \%, 70 \%, 80 \%$, and $90 \%)$ for 5 minutes each and in pure ethanol (100\%) for 10 minutes. The dehydrated specimens were dried by using a critical point dryer (Samdri795; Tousimis Research Corporation, Rockville, MD) and then mounted on SEM stubs and sputter-coated with gold-palladium (Hummer VI; Technic Inc, Anaheim, CA) under a constant current of $10 \mathrm{~mA}$ for 2 minutes. Finally, specimens were examined with SEM (Stereoscan 260; Cambridge Instruments, Cambridge, UK) at an accelerating voltage of $8-10 \mathrm{kV}$.

The cell viability data were analyzed statistically by using one-way analysis of variance (SPSS for Windows 11.0; SPSS, Chicago, IL) at a significance level of $P<.05$.

\section{Results \\ Cytotoxicity of the Set Cements}

Results of the flow cytometry assay on the cell viability of fibroblasts in the different concentrations of extracts derived from the set calcium silicate-based (Biodentine and MTA) and GIC materials are summarized in Figure 1. Cell viability depended on the type of material, culture medium, and incubation time that the cells were exposed to. After culturing for 1 day, cells incubated with extracts from Biodentine and MTA showed the highest viabilities at all extract concentrations, whereas cells exposed to GIC extracts displayed the lowest viabilities $(P<.001)$. After culturing for 3 days, there was no statistically significant difference in cell viabilities between Biodentine and MTA at all concentrations, GIC at extract concentration of 1:8, and the DMEM control $(P>.05)$. Cell viabilities of GIC at other concentrations (undiluted and 1:1, 1:2, and 1:4 dilutions) were significantly lower than the DMEM control $(P<.01)$. After culturing for 7 days, cell viabilities of undiluted extracts from Biodentine, MTA, and GIC were significantly lower than the DMEM control $(P<.01)$, but there was no significant difference between the cell viabilities of diluted extracts from Biodentine and MTA (1:1, 1:2, 1:4, and 1:8 dilutions), low concentration of 

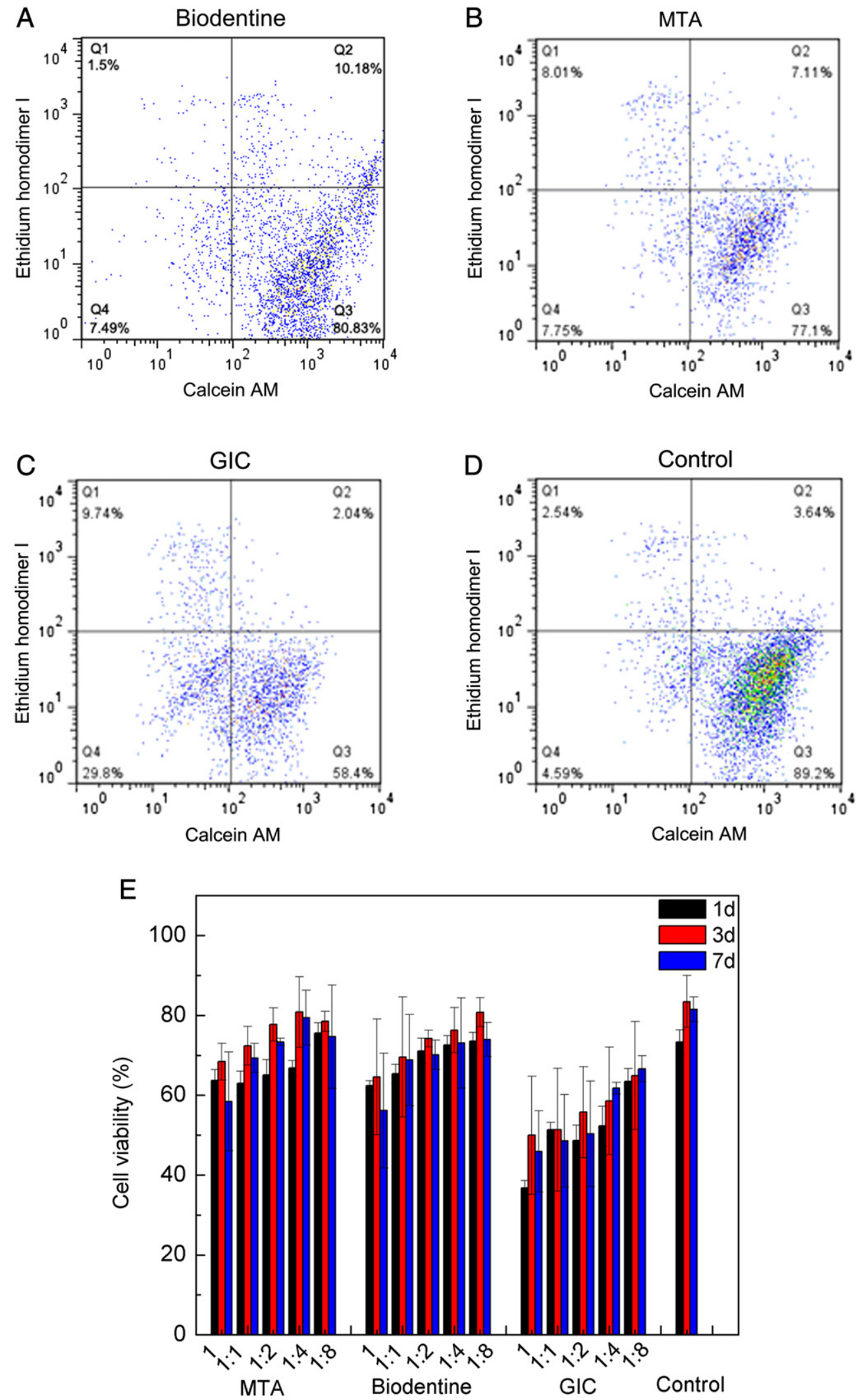

Figure 1. Representative 2-dimensional dot plots of the flow cytometry data derived from CAM and EthD-1 stained human gingival fibroblasts after exposure to extracts from $(A)$ Biodentine, $(B)$ ProRoot MTA, $(C)$ GIC Fuji IX, and $(D)$ DMEM control for culture for 7 days. The dot plot represents the distribution of viable (lower right), unstained cells (lower left), early apoptotic (upper right), and dead cells (upper left), respectively. (E) A histogram comparing the cell viability of extracts with various concentrations derived from Biodentine, MTA, GIC, and DMEM control after cell culture for 1, 3, and 7 days. The results show mean \pm standard deviation of 3 parallel experiments performed in triplicate. 

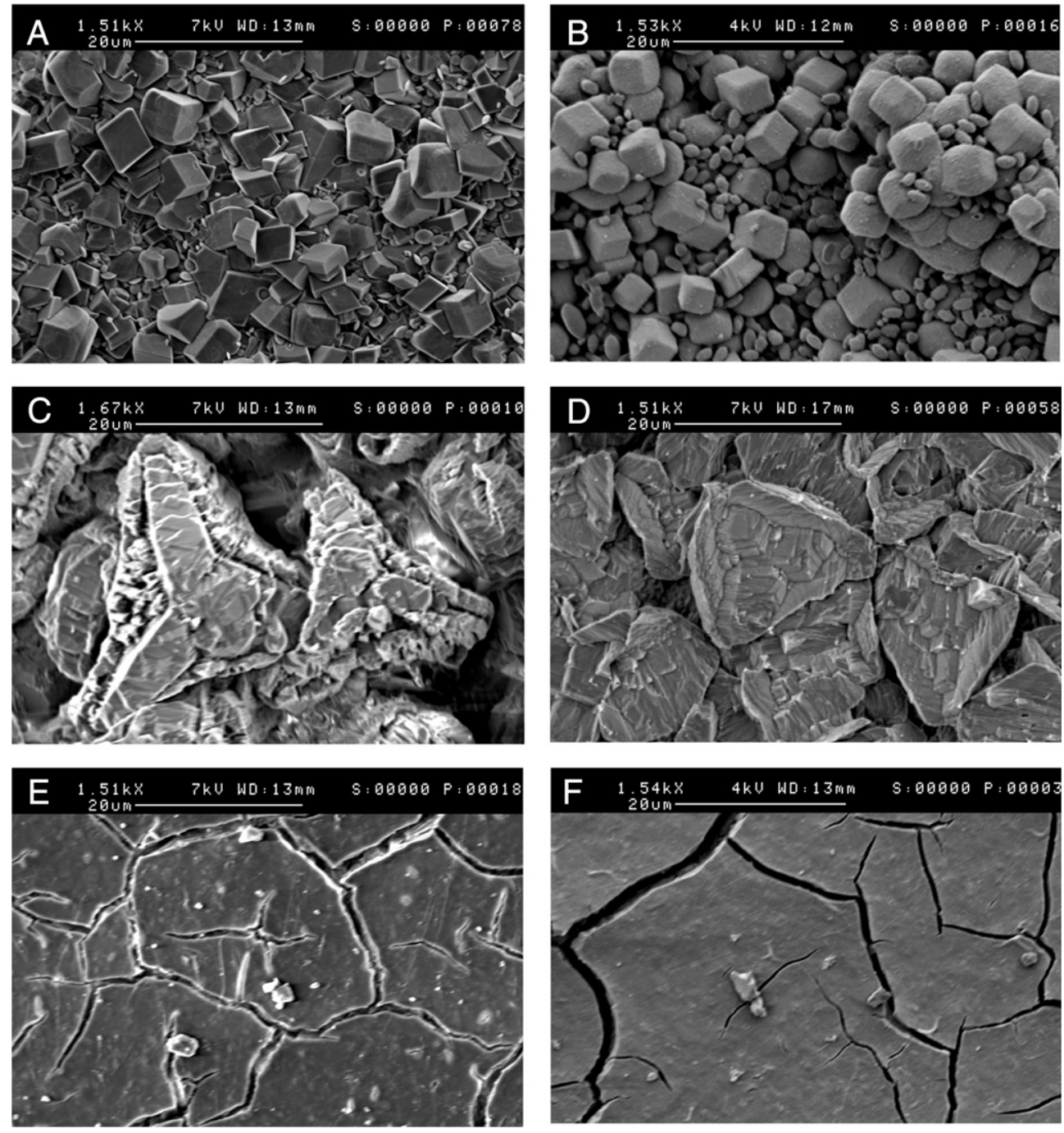

Figure 2. Scanning electron micrographs of the surface morphology of $(A$ and $B)$ Biodentine, $(C$ and $D)$ ProRoot MTA, and $(E$ and $F)$ GIC Fuji IX after immersion in distilled water for 5 days $(A, C$, and $E)$ or in DMEM culture medium $(B, D$, and $F)$ for 7 days.

extracts from GIC (1:8 dilution), and the DMEM control $(P>.05)$. Cell viabilities at high concentration of extracts (undiluted) were lower than those at low concentration of extracts (1:8 dilution) for all materials tested $(P<.05)$. There was no significant difference in cell viability among Biodentine, MTA, and GIC at low concentration extracts (1:8 dilution) and the DMEM control during the entire experimental period $(P>.05)$. There was no significant difference in cell viabilities between Biodentine and MTA at the same extract concentration during the entire experimental period $(P>.05)$.

\section{Cell Adhesion Assay}

Representative surfaces of the set Biodentine, MTA, and GIC after incubation in distilled water or DMEM culture medium are shown in Figure 2. Biodentine showed an uneven crystalline surface structure after incubation in water or DMEM (Fig. $2 A$ and $B$, respectively), whereas MTA showed the typical structure of calcium silicate hydrated gel on the surface of the crystals (Fig. $2 C$ and $D$ ). GIC displayed a smooth, noncrystallized surface (Fig. $2 E$ and $F$ ).

After culture for 1, 3, and 7 days on the material surfaces, fibroblasts exhibited different cell morphologies (Fig. 3). Gingival fibroblasts seeded on Biodentine and MTA surfaces showed similar characteristics; the fibroblasts attached to and spread over the material surface displaying the typical spindle-shaped fibroblast morphology after an overnight culture (Fig. $3 A$ and $D$ ). After culture for 3 days and 7 days, increased numbers of attached cells were seen in contact with each other on the surface of both Biodentine and MTA (Fig. $3 B, C, E$, and $F$ ). In the case of GIC, only a few poorly spread fibroblasts were observed after 1-day incubation, and some of the attached cells had a round shape (Fig. 3G) with vacuoles and blebs on their cell surface (insert in Fig. $3 G$ ). After both 3 days and 7 days of incubation (Fig. $3 H$ and $I$ ), fibroblasts showed a more spread cell morphology as compared with the earlier time point, but the cells were only sparsely distributed over the GIC surface and appeared to display only few cell-cell contacts.

\section{Discussion}

Materials used in endodontics should preferably be biocompatible. This is particularly desirable when they are placed in direct contact with living tissue such as in pulp capping, perforation repair, or when used as a retrograde filling. Biodentine, a new calcium silicate-based material, is designed to be placed in permanent and close contact with periradicular tissue, and therefore, it is important to assess its possible cytotoxic effects on human gingival fibroblasts. MTA, a calcium 

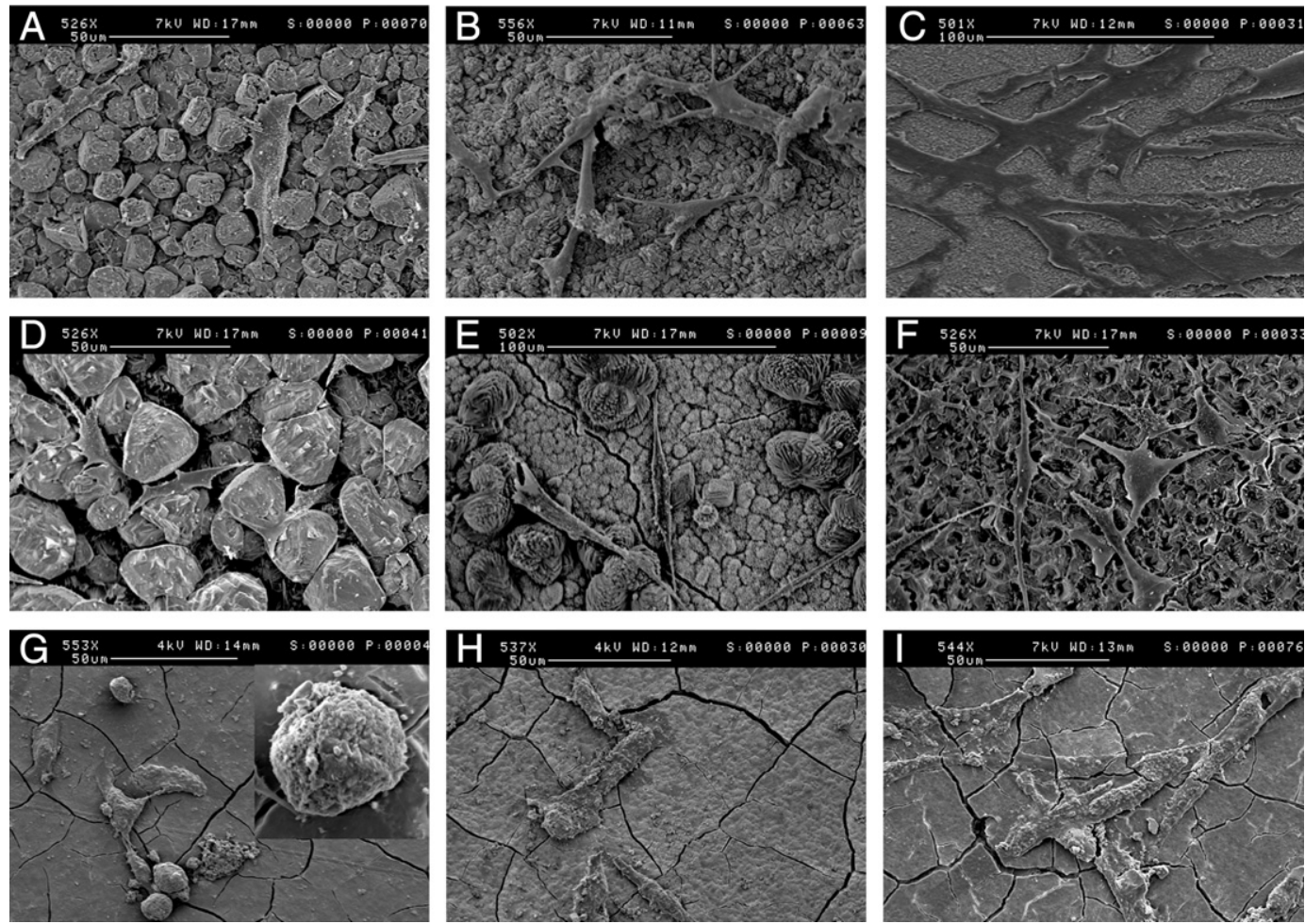

Figure 3. Scanning electron micrographs of the morphology of human gingival fibroblasts attached on the surface of Biodentine $(A-C)$, ProRoot MTA $(D-F)$, and GIC Fuji IX $(G-I)$ after culture in DMEM culture medium for 1 day $(A, D$, and $G), 3$ days $(B, E$, and $F)$, or 7 days $(C, F$, and $I)$. The $i n s e t$ in $(G)$ shows a high magnification image of a round-shaped cell.

silicate-based material, is the gold standard bioceramic cement that has been extensively studied and recognized as a bioactive and biocompatible material. Because of their adhesiveness and release of fluoride, glass ionomers have also gained popularity as the filling material in the treatment of cervical resorptions. Fuji IX is a conventional GIC that has a relatively high viscosity because of its fine grain powder and because it contains polyacrylic acid. Fuji IX GIC sets rapidly and is relatively resistant to early moisture, and its solubility to oral fluids is low (25). The characteristic of a short setting time of Fuji IX GIC is similar to Biodentine and may be an advantage in certain clinical situations such as perforation repair $(26,27)$. In the present study, the biocompatibility of ProRoot MTA and Fuji IX GIC was evaluated in comparison with Biodentine.

Several methods and strategies are available for cytotoxicity testing of materials, each with its merits and limitations. The methyl-thiazoltetrazolium (MTT) colorimetric assay is widely used as a standard assay for evaluating the cytotoxicity of new biomaterials and has been routinely used to test dental and endodontic filling materials in cell culture systems (28-30). However, MTT assays may underestimate cellular damage and detect cell death only at the stage of apoptosis when cellular metabolism is substantially reduced (14); these assays have poor linearity with the cell number and low sensitivity to environmental conditions $(31,32)$. To determine cell viability and cytotoxicity, alternative approaches have recently been proposed by using fluorescent dyes and flow cytometry (14), because this type of method is generally faster, less expensive, safer, and more sensitive to the cytotoxic events than alternative methods. In the present study, a 2-color flow cytometric cytotoxicity assay was used, which used 2 fluorescent stains (calcein AM [CAM] and EthD-1) to label live and dead cells. In this assay, the green fluorescent dye CAM was used to label live cells; this dye readily enters cells and is converted to calcein by intracellular esterase activity of live cells producing an intense uniform green fluorescence (excitation/emission $495 \mathrm{~nm} / 515 \mathrm{~nm}$ ). On the other hand, the red fluorescent dye EthD-1 was used to label dead cells; this type of dye enters cells with damaged membranes and binds to nucleic acids to produce a 40-fold enhancement of bright red fluorescence in the dead cells (excitation/emission $495 \mathrm{~nm} / 635 \mathrm{~nm}$ ). EthD-1 is excluded by the intact plasma membrane of live cells. Flow cytometer analyzes the stained cells with different fluorescent characteristics at a single cell level and assesses the relative population viability at the end of the assay (33). Similar to a previous work (14), in the present study, the proportion of viable cells out of the total population of cells present in the cultures was measured and statistically compared.

The degradation products and elution substances from endodontic cements might get access to periodontal tissues in several ways (34) and affect the healing processes, depending on their cytotoxicity. Therefore, extracts of various concentrations derived from Biodentine, MTA, and GIC were examined for cytotoxicity; these extracts can also simulate the postsurgical root-end environment where toxic elements of the retrofilling material leach into the surrounding fluids in the bony crypt (23, 35). A series of extracts of different concentrations were made to observe a possible dose-response relationship (23). In the present study, the viabilities of cells exposed to extracts derived from all the materials tested were highly dependent on extract concentration, and the viabilities of cells exposed to extracts from Biodentine and MTA at low extract concentration (1:8) showed no significant difference between each other and compared with the DMEM control.

Extracts from GIC Fuji IX caused significantly more cell death at all extract concentrations than extracts from Biodentine, MTA, and the DMEM control after culture for 1 day. This could probably be attributed to small amounts of aluminum and/or iron ions present in GIC extracts (36). It is generally assumed that leachable components of dental 
materials are contributing to adverse effects to cell cultures (37). Several in vitro studies have shown that the cytotoxicity of conventional GIC is lower than that of resin-modified GIC on cultured cells because the resin-modified GIC contains toxic leachable components. In a recent in vitro study, de Souza Costa et al (37) found that Fuji IX GP and KetacMolar were the least cytotoxic materials among the 5 tested GIC cements.

The surface topography of biomaterials is an important factor that determines cell interactions with the materials (38). Both Biodentine and MTA showed crystalline, uneven surface topography, whereas GIC surfaces appeared smooth. In general, a relatively smooth surface topography favors cell adhesion and growth (38). Therefore, it is likely that the poor initial spreading of fibroblasts on the GIC surfaces compared with Biodentine or MTA was caused by leaching of substances and/or other surface properties that adversely affect cell interactions with the material. However, some fibroblasts eventually spread and survived on the GIC surface after incubation for 7 days, suggesting that over time, cells can overcome a low cytotoxic effect of GIC Fuji IX.

\section{Conclusions}

Human gingival fibroblasts showed similar response to extracts from Biodentine and MTA as measured by cytotoxicity assay and cell growth on set materials.

\section{Acknowledgments}

The authors deny any conflicts of interest related to this study.

\section{References}

1. Laurent $\mathrm{P}$, Camps J, De Méo $\mathrm{M}$, et al. Induction of specific cell responses to a $\mathrm{Ca}(3)$ $\mathrm{SiO}$ (5)-based posterior restorative material. Dent Mater 2008;24:1486-94.

2. Torabinejad M, Watson TF, Pitt Ford TR. Sealing ability of a mineral trioxide aggregate when used as a root end filling material. J Endod 1993;19:591-5.

3. Rao A, Rao A, Shenoy R. Mineral trioxide aggregate: a review. J Clin Pediatr Dent 2009;34:1-7.

4. Parirokh M, Torabinejad M. Mineral trioxide aggregate: a comprehensive literature review_part I: chemical, physical, and antibacterial properties. J Endod 2010;36: $16-27$.

5. Torabinejad M, Parirokh M. Mineral trioxide aggregate: a comprehensive literature review_part II: leakage and biocompatibility investigations. J Endod 2010;36: 190-202.

6. Parirokh M, Torabinejad M. Mineral trioxide aggregate: a comprehensive literature review-part III: clinical applications, drawbacks, and mechanism of action J Endod 2010;36:400-13.

7. Darvell BW, Wu RC. "MTA": a hydraulic silicate cement-review update and setting reaction. Dent Mater 2011;27:407-22.

8. Camilleri J. Characterization and hydration kinetics of tricalcium silicate cement for use as a dental biomaterial. Dent Mater 2011;27:836-44

9. Camilleri J. Evaluation of the effect of intrinsic material properties and ambient conditions on the dimensional stability of white mineral trioxide aggregate and Portland cement. J Endod 2011;37:239-45.

10. Camilleri J. Characterization of hydration products of mineral trioxide aggregate. Int Endod J 2008;41:408-17.

11. Shahi S, Yavari HR, Rahimi S, et al. Comparison of the sealing ability of mineral trioxide aggregate and Portland cement used as root-end filling materials. J Oral Sci 2011;53:517-22.

12. El Sayed M, Saeed M. In vitro comparative study of sealing ability of Diadent BioAggregate and other root-end filling materials. J Conserv Dent 2012;15:249-52.
13. Sawyer AN, Nikonov SY, Pancio AK, et al. Effects of calcium silicate-based materials on the flexural properties of dentin. J Endod 2012;38:680-3.

14. Wei W, Qi YP, Nikonov SY, et al. Effects of an experimental calcium aluminosilicate cement on the viability of murine odontoblast-like cells. J Endod 2012;38:936-42.

15. Gomes Cornélio AL, Salles LP, Campos da Paz M, et al. Cytotoxicity of Portland cement with different radiopacifying agents: a cell death study. J Endod 2011;37: 203-10.

16. Damas BA, Wheater MA, Bringas JS, Hoen MM. Cytotoxicity comparison of mineral trioxide aggregates and EndoSequence bioceramic root repair materials. J Endod 2011;37:372-5.

17. Bodrumlu E. Biocompatibility of retrograde root filling materials: a review. Aust Endod J 2008;34:30-5.

18. Asgary S, Eghbal MJ, Parirokh M. Sealing ability of a novel endodontic cement as a root-end filling material. J Biomed Mater Res A 2008;87:706-9.

19. Zanini M, Sautier JM, Berdal A, Simon S. Biodentine induces immortalized murine pulp cell differentiation into odontoblast-like cells and stimulates biomineralization. J Endod 2012;38:1220-6.

20. Raskin A, Eschrich G, Dejou J, About I. In vitro microleakage of Biodentine as a dentin substitute compared to Fuji II LC in cervical lining restorations. J Adhes Dent 2012;14:535-42.

21. Leiendecker AP, Qi YP, Sawyer AN, et al. Effects of calcium silicate-based materials on collagen matrix integrity of mineralized dentin. J Endod 2012;38: 829-33.

22. Laurent $\mathrm{P}$, Camps J, About I. Biodentine(TM) induces TGF- $\beta 1$ release from human pulp cells and early dental pulp mineralization. Int Endod J 2012;45:439-48.

23. Ma J, Shen Y, Stojicic S, Haapasalo M. Biocompatibility of two novel root repair materials. J Endod 2011;37:793-8.

24. International Organization for Standardization. Biological Evaluation of Medical Devices: Part 5-Tests for Cytotoxicity: In Vitro Methods, ISO 10993. Geneva, Switzerland: International Organization for Standardization; 1992.

25. Nagaraja Upadhya P, Kishore G. Glass ionomer cement: the different generations. Trends Biomater Artif Organs 2005;18:158-65.

26. Goldberg M, Pradelle-Plasse N, Tran XV, Colon P. Emerging trends in (bio)material research. In: Goldberg M, ed. Biocompatibility or Cytotoxic Effects of Dental Composites. Oxford, UK: Coxmoor Publishing Company; 2009:181-203.

27. GC Europe. Fuji IX GP and Fuji IX GP Fast. Available at: http://www.gceurope.com/ pid/4/leaflet/en_Leaflet.pdf. Accessed September 2, 2012.

28. Gorduysus M, Avcu N, Gorduysus 0, et al. Cytotoxic effects of four different endodontic materials in human periodontal ligament fibroblasts. J Endod 2007; 33:1450-4.

29. Jafarnia B, Jiang J, He J, et al. Evaluation of cytotoxicity of MTA employing various additives. Oral Surg Oral Med Oral Pathol Oral Radiol Endod 2009;107:739-44.

30. Hirschman WR, Wheater MA, Bringas JS, Hoen MM. Cytotoxicity comparison of three current direct pulp-capping agents with a new bioceramic root repair putty. J Endod 2012;38:385-8.

31. Haselsberger K, Peterson DC, Thomas DGT, Darling JL. Assay of anticancer drugs in tissue culture: comparison of a tetrazolium-based assay and a protein binding dye assay in short-term cultures derived from human malignant glioma. Anti-Cancer Drugs 1996;7:331-8

32. Marqués-Gallego $\mathrm{P}$, den Dulk $\mathrm{H}$, Backendorf $\mathrm{C}$, et al. Accurate non-invasive image-based cytotoxicity assays for cultured cells. BMC Biotechnology 2010;10: 43-50.

33. Papadopoulos NG, Dedoussis GV, Spanakos G, et al. An improved fluorescence assay for the determination of lymphocyte-mediated cytotoxicity using flow cytometry. J Immunol Methods 1994;177:101-11.

34. Huang TH, Ding SJ, Hsu TZ, et al. Root canal sealers induce cytotoxicity and necrosis. J Mater Sci Mater Med 2004;15:767-71.

35. Keiser K, Johnson CC, Tipton DA. Cytotoxicity of mineral trioxide aggregate using human periodontal ligament fibroblasts. J Endod 2000;26:288-91.

36. Soheili Majd E, Goldberg M, Stanislawski L. In vitro effects of ascorbate and Trolox on the biocompatibility of dental restorative materials. Biomaterials 2003;24:3-9.

37. de Souza Costa CA, Hebling J, Garcia-Godoy F, Hanks CT. In vitro cytotoxicity of five glass-ionomer cements. Biomaterials 2003;24:3853-8.

38. Bacakova L, Filova E, Parizek M, et al. Modulation of cell adhesion, proliferation and differentiation on materials designed for body implants. Biotechnol Adv 2011;29: 739-67. 